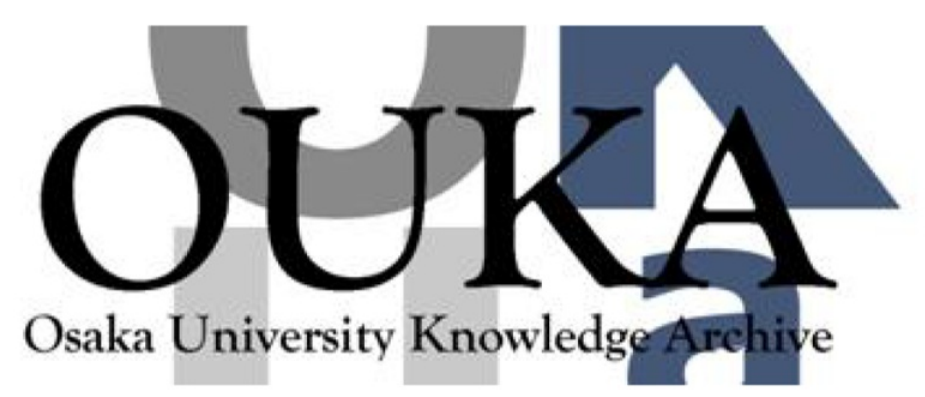

\begin{tabular}{|c|c|}
\hline Title & $\begin{array}{l}\text { Performance comparison of self-focusing with } \\
1053-\text { and } 351-n m \text { laser pulses }\end{array}$ \\
\hline Author (s) & Tanaka, K.A.; Hashimoto, H.; Kodama, R. et al. \\
\hline Citation & Physical Review E. 60(3) p.3283-p.3288 \\
\hline Issue Date & $1999-09$ \\
\hline oaire:version & VoR \\
\hline URL & https://hdl. handle. net/11094/3083 \\
\hline rights & $\begin{array}{l}\text { Tanaka, K.A., Hashimoto, H., Kodama, R., Mima, } \\
\text { K., Sentoku, Y., Takahashi, K. "Physical Review } \\
\text { E, 60, 3, 3283-3288, 1999-09. "Copyright } 1999 \\
\text { by the American Physical Society." }\end{array}$ \\
\hline Note & \\
\hline
\end{tabular}

Osaka University Knowledge Archive : OUKA

https://ir. Library. osaka-u. ac. jp/

0saka University 


\title{
Performance comparison of self-focusing with 1053- and 351-nm laser pulses
}

\author{
K. A. Tanaka, ${ }^{*}$ H. Hashimoto, R. Kodama, K. Mima, Y. Sentoku, and K. Takahashi \\ Institute of Laser Engineering, Osaka University Yamada-Oka 2-6, Suita, Osaka 565 Japan
}

(Received 2 February 1999)

\begin{abstract}
Hole boring characteristics of laser beams are studied using two different laser wavelengths in preformed plasmas with overdense regions. We have shown that a whole beam self-focusing is created in plasma with a considerable density scale length using a $1 \mu \mathrm{m}$ wavelength laser. The whole beam self-focusing of this type could be used for guiding the ultrahigh intense laser pulse to a highly compressed core for studying the feasibility of a fast ignitor. There is a clear difference in the hole-boring characteristics between two laser wavelengths at 1053 and $351 \mathrm{~nm}$, both in the experiment and the simulation. Using the third-harmonic laser, a whole beam self-focusing is never created. The 351-nm laser beam broke up into filaments resulting in plasma jets observed in our interferogram. [S1063-651X(99)06109-7]

PACS number(s): 52.35.Mw, 52.40.Nk, 52.65.Rr
\end{abstract}

The self-focusing and filamentation of a laser beam have attracted a lot of attention and have been studied extensively for improvement of the uniformity of direct or indirect drive implosions [1,2], applications to soft $\mathrm{x}$-ray lasers $[3,4]$, the generation of high harmonics [5], and the fast ignitor concept [6]. The self-focusing of a laser pulse with a duration on the order of 10-100 ps with a considerable energy could play an important role during predrilling or hole boring through the surrounding plasma at close to the final stages of the implosion in fast ignition. If this predrilling could be controlled accurately, a subsequent ultrahigh intense laser pulse could be guided in the predrilled, self-focused channel with minimum energy dissipation to reach imploded cores for the fast ignition. Whole beam self-focusing, in which the ponderomotive effect dominates, has been studied in detail in underdense plasmas, using a $1-\mu \mathrm{m}$ laser beam with both cylinder and line focus at below $5 \times 10^{16} \mathrm{~W} / \mathrm{cm}^{2}$ [7]. Relativistic selffocusing has been studied in numerical simulations [8] and it has been predicted that the beam tends to break into filaments at first, but penetrates later into an overdense region as a whole beam. Relativistic self-focusing with a 0.6 ps pulse width and a few hundred $\mathrm{mJ}$ energy has been observed in a uniform plasma [9]. Self-focusing in a ponderomotive selffocusing laser intensity region with a $100 \mathrm{ps}$ and a $100 \mathrm{~J}$ energy has not been observed using a beam with a circular cross section directly with any probe laser beam in an exponential plasma profile with an overdense region. In addition, the dependence of the self-focusing on the laser wavelengths is not fully understood when the ponderomotive force is dominant. Self-focusing due to the ponderomotive force could be of critical importance, since a long distance selffocusing could be needed to guide an ultraintense laser pulse for fast ignitor ignition [6].

\footnotetext{
*Author to whom correspondence should be addressed. Address correspondence to Institute of Laser Engineering, Osaka University Suita, Osaka 565, Japan. Also at the Department of Electromagnetic Energy Engineering, Osaka University, Yamada Oka 2-1, Suita, Osaka 565, Japan. FAX: +81-6-6877-4799. Electronic address: katanaka@ile.osaka-u.ac.jp
}

In this paper, the 1053- and 351-nm lasers hole boring characteristics are studied, using $100 \mathrm{ps}$ laser pulses at up to $2 \times 10^{17} \mathrm{~W} / \mathrm{cm}^{2}$ laser intensities. The hole boring lasers are injected into preformed plasmas with overdense regions created on massive plastic targets. The 1053-nm laser beam clearly shows a whole beam self-focusing in an underdense plasmas, while the 351-nm beam shows many striations at the same laser intensities. Using a 2(1/2) D PIC code we compare the performance of two laser wavelengths keeping the peak laser power the same as the experiments. The simulation's results are consistent with the experiment.

Experiments were carried out using the GEKKO XII laser beam system at the Institute of Laser Engineering, Osaka University. One of the horizontal beams was chosen for a hole-boring beam with a $1053 \mathrm{~nm}$ wavelength, while two beams at a third harmonic laser wavelength $(\lambda=351 \mathrm{~nm})$ were focused obliquely at $50^{\circ}$ angle on target with a $300 \mu \mathrm{m}$ spot diameters at a $10^{14} \mathrm{~W} / \mathrm{cm}^{2}$ intensity. Similarly, another horizontal beam was used to study the third-harmonic drilling performance with additional two $351 \mathrm{~nm}$ laser beams for preforming plasmas. For all the beams the $f$ number of focus lens was 3 . The laser pulse width was 100 ps full width at half maximum (FWHM). Both infrared and blue hole-boring pulses can be focused within a $30 \mu \mathrm{m}$ spot size, resulting in $2 \times 10^{17} \mathrm{~W} / \mathrm{cm}^{2}$ irradiation intensity with $100 \mathrm{~J}$ energy. The spot size is determined by the optical aberrations integrated in the glass laser system. The focal spot intensity has a Gaussian spatial distribution at the best focus. Time delay between the preformed plasma formation and the hole boring pulses is typically $1 \mathrm{~ns}$. Targets were 200 and $100 \mu \mathrm{m}$ thick plastic planes. The performance of hole boring was studied changing the focusing positions of the drilling laser beam from 0 to $450 \mu \mathrm{m}$ from the original target surface. Focusing positions from 200 to $250 \mu \mathrm{m}$ produced whole beam selffocusing with the 1053-nm hole boring laser under the preformed plasma conditions. This whole beam self-focusing was discussed in Ref. [11], where we measured x-ray pinhole images and backscattered light temporally resolved spectrum. The x-ray image showed a $30 \mu \mathrm{m}$ emission spot of the self-focused beams at the target surface, while the laser beam diameter is $80 \mu \mathrm{m}$ in vacuum at the target surface with a condition same with this experiment (See Fig. 4 in Ref. 


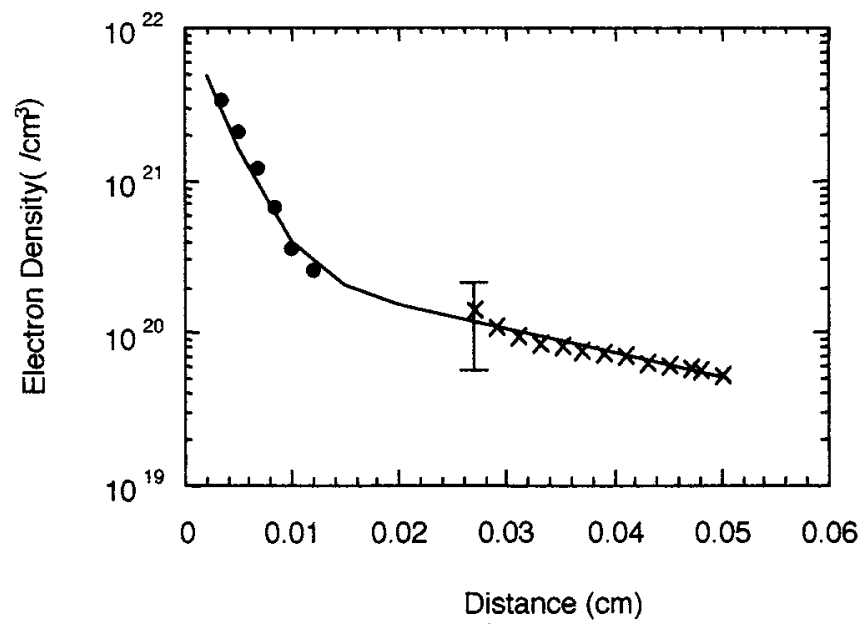

FIG. 1. Preformed plasma density profile. The profile was measured by both x-ray laser (circle) [10] and uv laser (cross) probe systems in the experiment. Circle data points show the profile measured by the x-ray laser and the cross by the uv probe.

[11]). The backscattered light spectrum showed a clear Doppler shift, indicating that the drilling speed into the plasma is $7 \times 10^{7} \mathrm{~cm} / \mathrm{s}$. This drilling speed was consistent with the self-focused laser intensity $10^{18} \mathrm{~W} / \mathrm{cm}^{2}$ instead of the initial focused intensity $10^{17} \mathrm{~W} / \mathrm{cm}^{2}$. Under these experimental conditions, the self-focusing appeared to propagate over a 70 $\mu \mathrm{m}$ distance in the overdense plasma region keeping a very tight spot of $30 \mu \mathrm{m}$ or less and drilling well into the overdense regions up to very close to the original solid density through a pre-formed plasma.

An ultraviolet $(\lambda=263 \mathrm{~nm})$ probe pulse of a $10 \mathrm{ps}$ (FWHM) was introduced to set up an image interferometer system at the experimental chamber. A synchronized probe pulse, split off at the system oscillator is transported through a glass fiber to a regenerative amplifier and pulse compression grating section located close to the experimental chamber. The probe pulse was frequency quadrupled with KDP crystals to be a $263-\mathrm{nm}$ wavelength at typically $100 \mu \mathrm{J}$ with a $1 \mathrm{~cm}$ diameter beam. The interferogram was imaged on a uv sensitive CCD camera with a $5 \mu \mathrm{m}$ spatial resolution. The uv probe laser observes the hole-boring behavior normal to the drilling beam with a 200 ps delay relative to the drilling. The timing is chosen so as not to miss the drilling within the systematic jitter between the probe and main laser beams. Exact timing has been monitored for each shot. The maximum plasma density for the UV interferometer system is $3-5 \times 10^{20}$ electron density $\left(n_{e}\right)$ due to the uv probe ray refraction through the preformed plasma, though the cutoff density of this probe laser light is $1.6 \times 10^{22}$. Shown in Fig. 1 is the result of preformed plasma density scale length measurement using this UV probe system. Obtained interferogram pictures are reduced to density information using an Abel inversion technique [2]. The density scale length of the preformed plasma shown in Fig. 1 is about $40 \mu \mathrm{m}$ at around the cutoff density $\left(n_{c}\right)$, which has been measured by both $\mathrm{x}$-ray [10] and UV laser probe beams during the experiment. In the figure the data points (circles) around the critical density are obtained with the $\mathrm{x}$-ray probe system and the ones (crosses) below the critical density are obtained with the UV probe system. The positions of the $n_{c}$ surface is at from 70 to $100 \mu \mathrm{m}, n_{c} / 10$ is at $300 \mu \mathrm{m}$ and $4 \times 10^{19} / \mathrm{cm}^{3}$ was at $500 \mu \mathrm{m}$ from the original target surface.

Typical interferograms are shown in Fig. 2 and Fig. 4. Those are taken for such hole-boring shots into the preformed plasma (Fig. 1) with 1053-nm and 351-nm laser at a focused intensity $10^{17} \mathrm{~W} / \mathrm{cm}^{2}$, respectively. In Fig. 2(a), the interferogram picture is shown of whole beam self-focusing at $1053-\mathrm{nm}$ wavelength. The whole beam self-focusing was observed on this shot and was confirmed from the x-ray pinhole pictures very similar to the ones of Ref. [11]. We see interferogram fringes form a spatial cone as marked in Fig. 2(b). The ridges of the spatial cone follow the lens-focusing cone $(f / 3)$ in the very underdense region. The interferogram fringes of the preformed plasma also seem to be flattened normal to the laser incident axis. In the picture, a highdensity region bends the interferogram fringes toward the vacuum side because of the phase characteristics. The diameters of these flattened regions become smaller toward the target surface. It is also possible to see that interferogram fringes at around the ridges bend toward the underdense side with a small curvature. All these features indicate that a cylindrically symmetric plasma cone is created in the plasma. The plasma cone has density higher than the background due

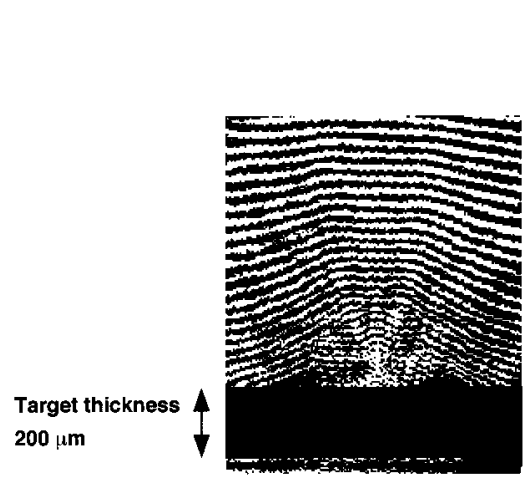

(a)

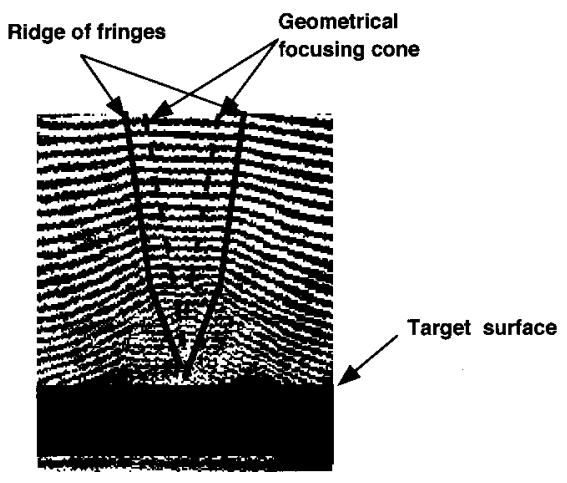

(b)

FIG. 2. uv interferometer image of 1053-nm laser hole boring. The image was taken with a 263-nm, 10 ps probe pulse. (a) and (b) are same except that there are two kinds of lines overlapped to enhance the details of the picture. The dotted lines show the geometrical cone of $f=3$. The line shows the ridge of the interference patterns where a cylindrical cone formed during the laser beam self-focusing. The focusing degree appears to become more rapid $350 \mu \mathrm{m}$ from the target surface, indicative of convective whole beam self-focusing. 


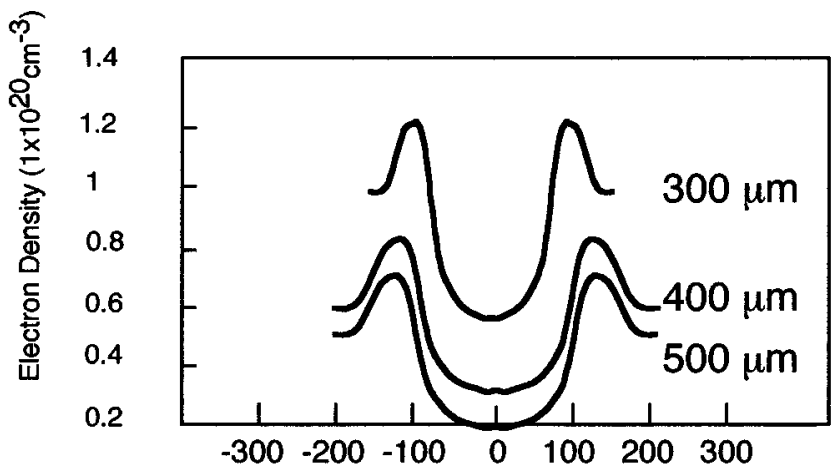

Radius ( $\mu \mathrm{m})$

FIG. 3. Density profiles normal to the laser axis as a parameter of axial distance from the original target surface. Density information is obtained from the interferogram picture using a technique the same as the one used for Fig. 1. The cross sections were shown at 300,400 , and $500 \mu \mathrm{m}$ from the target surface.

to the ponderomotive effect of the laser beam. Then the cone angle becomes large toward the target. At $350 \mu \mathrm{m}$ or closer to the target, the 1053-nm drilling appeared to focus more rapidly with, say, an $f / 1.7$ compared to the $f / 3$ cone given by the focus lens. We believe that laser starts self-focusing convectively from this point along the incident axis. The best focus position was set at $210 \mu \mathrm{m}$ from the original target surface. Density profiles reduced from the data in Fig. 2 are shown in Fig. 3. The cross sections are shown at 300, 400, and $500 \mu \mathrm{m}$ from the target surface. It is estimated that the density hole at the center and the maximum wall electron densities are $6 \times 10^{19}$ and $1.2 \times 10^{20} / \mathrm{cm}^{3}$, respectively at 300 $\mu \mathrm{m}$ from the target surface in Fig. 3. The width of the hole was about $100 \mu \mathrm{m}$ at $200 \mu \mathrm{m}$ from the target. The width appears to be a little wide compared to the $30 \mu \mathrm{m}$ focal spot. We consider that the observed timing was 200 ps after the predrilling creation and the cylindrical plasma cone should expand radially until the force exerted by the laser and ambient plasma pressure balance. X-ray pinhole pictures for these whole beam self-focusing shots show a clear tight (30 $\mu \mathrm{m}$ diameter) emission from the surface of the plane target. This indicates that the whole beam self-focus penetrated the preformed plasma well into the overdense region very similar to Fig. 4 in Ref. [11]. Figures 2 and 3 show that there is a cylindrical plasma cone formed along the laser-focusing cone in the underdense plasma. The formed plasma cone shows rapid laser focusing when the laser light propagates closer to the target. A circularly focused laser changes transitively to a whole beam self-focusing in a plasma with a 100 ps pulse width and $100 \mathrm{~J}$ laser energy, and at a laser intensity $10^{17} \mathrm{~W} / \mathrm{cm}^{2}$. Considering that the ponderomotive force starts dominating at above $10^{16} \mathrm{~W} / \mathrm{cm}^{2}$, exceeding the plasma pressure, and the relativistic effect comes in at an intensity much higher than $10^{18} \mathrm{~W} / \mathrm{cm}^{2}$, this turn on of self-focusing is due to the ponderomotive force of the laser light $[12,13]$. For these shots, the predrilling speeds were monitored with the backscattered spectrum of the second-harmonic light. The Doppler shift in the backscattered spectrum reflects the cutoff density speed, indicating that the drilling speeds are about $1 \times 10^{8} \mathrm{~cm} / \mathrm{s}$, consistent with our previous measurement in Ref. [11].

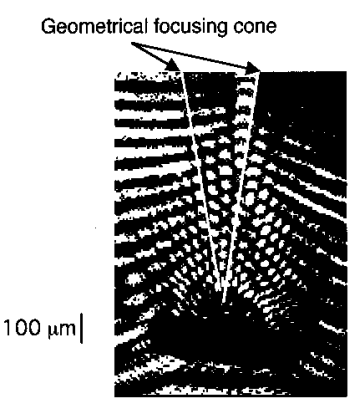

(a)

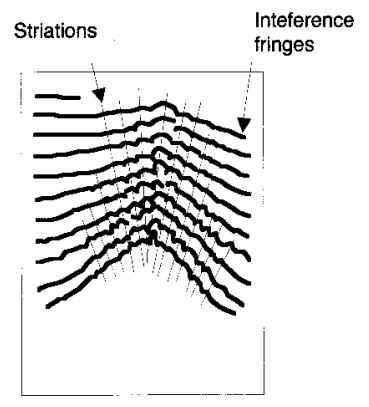

(b)
FIG. 4. (a) uv interferometer image of 351-nm laser case. The image shows that there are many striations in the underdense region. (b) The interference fringes of (a) are shown. The fine lines indicate that there are many striations in the underdense plasma. Considering the interfered fringe shift directions, the striations actually indicate high density jets.

When we use a 351-nm wavelength laser as a hole-boring beam, the observed interferogram image was strikingly different as shown in Fig. 4. Figure 4(a) shows the interferogram, which indicates many striations in the underdense region. The white lines show the focused laser geometrical cone. In Fig. 4(b) interference fringes are extracted out of Fig. 4(a). Fine lines indicates striations seen as the ridges of fringes. In Fig. 4, the 351-nm laser beam was focused at 100 $\mu \mathrm{m}$ away from the original target surface in the preformed plasma, which is the same as the one in Figs. 2 and 3. The focusing point was set for the 351-nm laser with the cutoff density ten times higher than the $1054 \mathrm{~nm}$ to have an equivalent effect on the plasma, though the focusing positions $d$ $=0,200$, and $400 \mu \mathrm{m}$ were all tested and the results were the same as shown in Fig. 4. The shot-to-shot reproducibility was good. One can see that the image seems to be dominated by many striations in the underdense area. Those striations appear normal to a preformed plasma surface. The target thickness was $100 \mu \mathrm{m}$ on this shot compared to the $200 \mu \mathrm{m}$ thick target used in Fig. 2. Knowing the phase shift directions along the striations, we could tell that those striations have a higher density than the background and are actually high-density jets. At $300 \mu \mathrm{m}$ from the target surface the fringe in a typical striation is shifted by half the fringe interval seen in this region. Considering that the striation size is typically $30 \mu \mathrm{m}$ width and that the plasma density is 4 $\times 10^{19} / \mathrm{cm}^{3}$, the density in the striation is $20 \%$ higher than the background plasma density. X-ray pinhole pictures on these shots show that there is only $\mathrm{x}$-ray emissions from the preformed plasma within the $300 \mu \mathrm{m}$ and no signs of hot spots, which could cause high density jets, if there was any. Thus we consider that the density jets seen in Fig. 4 are created either in a plasma region far away from the target surface and/or the temperature of the striated plasma is very low, resulting in that no striations are observed in the x-ray pictures.

Simulations from our 2(1/2)-dimensional particle code are compared to the experimental results. As the simulation setting, linear plasma density profiles are set from zero to $2 n_{c}$ along the $x$ direction for both infrared and uv laser runs. The preformed plasma consists of fully ionized deuterons. The initial electron and ion temperatures are $10 \mathrm{keV}$ and $100 \mathrm{eV}$. 


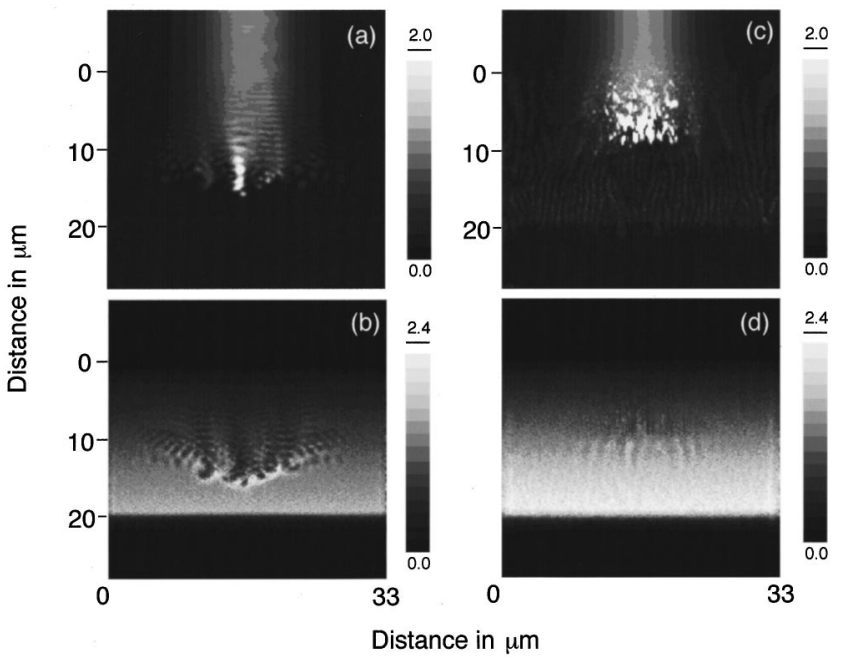

FIG. 5. (a) 2(1/2) PIC simulation result of 1053-nm laser selffocusing. The figure indicates the laser field intensity. After the beam tended to break up into three components, the laser beam merges into a whole beam self-focusing. (b) PIC simulation result for 1053-nm laser self-focusing. The figure indicates the electron density distribution for Fig. 2(a). According to the laser field intensity, the electron density distribution shows that a hole is bored. (c) 1(1/2) PIC simulation result of the 351-nm laser irradiation case. The figure indicates the laser field intensity. The laser breaks up into to many filaments in the underdense region and never goes beyond the critical density. (d) PIC simulation result for the 351-nm laser. The figure indicates the electron density distribution for Fig. 2(c). The figure indicates that many fine holes are created according to the fine laser filaments.

The density scale length is $20 \mu \mathrm{m}$. A laser power $2 \mathrm{TW}$ is focused within a $12 \mu \mathrm{m}$ spot diameter, resulting in the laser intensity $2 \times 10^{18} \mathrm{~W} / \mathrm{cm}^{2}$. The peak power in the simulation is chosen same as the experiment because of the simulation capacity. This can be rationalized since the occurrence of ponderomotive and relativistic self-focusing depends on laser power, not focused laser intensity [8]. As is well known, the critical power is determined by the competition of ponderomotive self-focusing and defocusing due to the diffraction in the ponderomotive regime and relativistic selffocusing and defocusing due to the diffraction in the relativistic regime. The focused intensity is ten times higher than the experiment in simulation. The laser intensity dependence is checked with the simulation at which laser intensity the laser beams start self-focusing (and has been confirmed to be consistent with the rational) and is shown later in Fig. 6 . The intensity has a Gaussian spatial profile. The highest plasma densities are $2.23 \times 10^{21}$ and $2.23 \times 10^{22} \mathrm{~cm}^{3}$ (two times the cut off densities) for the 1053-nm and 351-nm wavelength lasers. The $y$ direction perpendicular to the laserpropagating axis is periodic for the fields and particles. The $P$-polarized laser penetrates into the performed plasma from $x=0$ and is absorbed at the longitudinal boundaries, while the particles are simply reflected. We used $512 \times 512$ cells as a spatial mesh and 9216000 particles for electrons and ions (ion mass is $3680 m_{e}$ ).

The behavior of the laser penetration is shown in Fig. 5 . The field intensity and the electron plasma density are shown in Figs. 5(a) and 5(b) for the 1053-nm laser. Similarly, the

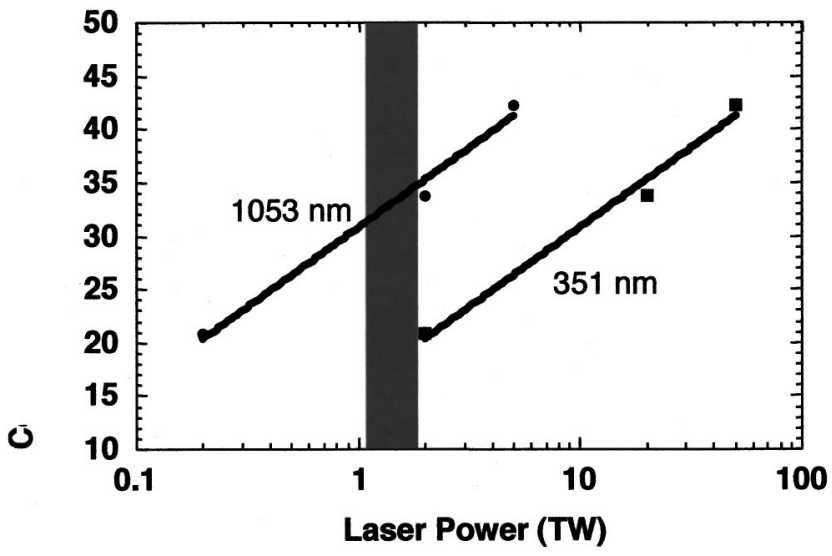

FIG. 6. Self-focus dependence on laser power for the 1053-nm and 351-nm laser light. Self-focusing characteristics are defined as the laser energy concentration fraction within a $3 \mu \mathrm{m}$ spot from the cutoff density to the head of the self-focusing at a time 1 ps. $25 \%$ is the original fraction contained in the Gaussian distribution beam on the vertical axis. Any value higher than this indicates a whole beam self-focusing. The 1053-nm case indicates a whole beam selffocusing starting at 1-2 TW laser power, while the 351-nm requires at least ten times more laser power. The shaded area shows laser power area used in the experiment.

field intensity and the electron plasma density are shown for the 351-nm case in Figs. 5(c) and 5(d). The field intensity in the 1053-nm case [Fig. 5(a)] shows that the laser penetrates into the performed plasma and tends to break up into three parts, but merges to a whole beam self-focusing. The whole beam self-focusing intensity becomes strong enough to go beyond the cutoff density. A single channel is formed and the laser propagation is stable all the way to the highest density after this time step. The penetration speed observed in the simulation is $1 / 100$ of the light speed at a self-focused intensity $2 \times 10^{18} \mathrm{~W} / \mathrm{cm}^{2}$. This value is close to 1 $\times 10^{8} \mathrm{~cm} / \mathrm{s}$, which has been estimated from the Doppler shift of the backscattered light spectrum in the previously reported experiment [11]. This self-focusing behavior shows a good agreement with our experimental observation.

In this simulation run, the maximum laser intensity is further increased from the initial $2 \times 10^{18} \mathrm{~W} / \mathrm{cm}^{2}$ to 6 $\times 10^{18} \mathrm{~W} / \mathrm{cm}^{2}$ because of the self-focusing effect. The laser light experiences some absorption along the filament wall during the propagation. The electron density distribution shows holes corresponding to the strong laser field as shown in Fig. 5(b). The ion movement is checked to show density distribution almost the same as the electrons.

The 351-nm laser run shows totally different results from the infrared. As soon as the 351-nm laser comes in the preformed plasma region, the laser beam tends to break up into many filaments and the most of the filaments are stopped at the cutoff density as shown in Fig. 5(c). The plasma density of Fig. 5(d) also shows that the many fine holes correspond to the filaments and do not extend beyond the cutoff density.

To test the self-focusing dependence on the laser power, we ran the simulation code for both laser wavelengths. The results are shown in Fig. 6. This graph shows those selffocusing characteristics versus laser power for the two laser wavelengths. The self-focusing characteristic is defined as 
the fractional laser energy concentrated within the $3 \mu \mathrm{m}$ spot from the cutoff density to the head of the self-focusing at a time 1 ps. Within the Gaussian beam spot, the fraction is $25 \%$ at first. The fraction higher than this value indicates a whole beam self-focusing. The shaded area in Fig. 6 corresponds to the laser power used in the experiment. The laser energy concentration becomes apparent at above 1-2 TW laser power for the 1053-nm laser. The whole beam selffocusing occurs for the infrared and blue at 0.4 and $4 \mathrm{TW}$, respectively. At $0.2 \mathrm{TW}$ for the infrared, the laser beam breaks up in the underdense plasma into filaments and ends up with a concentration fraction lower than the initial $25 \%$ value. 1-2 TW laser power is needed for sufficient selffocusing for the 1053-nm laser, consistent with the experimental result shown in Fig. 2. The 351-nm case shows that at least one order of magnitude higher laser power is needed to obtain the result same as the 1053-nm laser. At 1-2 TW laser power, the 351-nm laser showed filamentation [Fig. $1(b)]$ and the fraction becomes lower than the initial fraction. This difference observed in the 2D simulation for different wavelengths could be due to the ponderomotive force effect, which scales as $I_{L} \lambda_{L}^{2}$, where the $\lambda_{L}$ is the laser wavelength. It is interesting to discuss the beam breaking up into filaments in the simulation and many density jet striations appearing for the third harmonic. A growth for convective instabilities such as self-focusing is controlled by the imaginary part $k_{\operatorname{Im}}$ of $k$ : the wave number times the distance. The number of exponentiations for the instability is then $k_{\operatorname{Im}} x$ where $x$ is the propagation distance. Since the wave number $k$ is proportional to $1 / \lambda, k_{\operatorname{Im}} x$ is a function of $x / \lambda$. From this it is clear that the shorter wavelength laser experiences more convective growth over a given length. In the simulations, the setup conditions are scaleable from 351-nm to the 1053-nm laser based on this argument. Namely, a run performed in a $20 \mu \mathrm{m}$ spatial scale extent for the 351-nm laser corresponds to a $60 \mu \mathrm{m}$ extent for the 1053-nm laser. This indicates that the third-harmonic laser light propagates in a plasma effectively three times longer than the one for the 1053-nm laser and may possibly be affected by such a convective instability as filamentation, once the laser intensity is beyond the instability thresholds. We find that the thirdharmonic light starts to self-focus as a whole (see Fig. 6) at a laser intensity ten times higher than the infrared. This is consistent with the ponderomotive force scaling on the laser wavelength. The instability thresholds are estimated to be of the order of $10^{14} \mathrm{~W} / \mathrm{cm}^{2}$, and are all well exceeded in the experimental, Fig. 2, and the simulation, Fig. 5.

In Figs. 2 and 5, 1053-nm laser irradiation shows a clear indication of self-focusing. The intensity at this point is estimated to be $2 \times 10^{16} \mathrm{~W} / \mathrm{cm}^{2}$ in vacuum, well above the ponderomotive self-focusing $[14,15]$, and the smaller $f$ number cone is indicative of rapid laser focus. The self-focusing appeared to start $350 \mu \mathrm{m}$ from the target surface. The focusing distance may be expressed analytically as [2],

$$
L_{\mathrm{sf}}=0.8 r_{0}\left(n_{c} T_{\mathrm{keV}}\right)^{1 / 2} /\left(n_{e} I_{16} \lambda_{\mu \mathrm{m}}^{2}\right)^{1 / 2}
$$

Here $r_{0}$ is the focal spot radius, $T_{\mathrm{keV}}$ is the plasma temperature, $I_{16} \lambda_{\mu \mathrm{m}}^{2}$ is the laser intensity $\left(\times 10^{16} \mathrm{~W} / \mathrm{cm}^{2}\right)$ times the laser wavelength squared in $\mu \mathrm{m}$. From the observed param- eters of $r_{0}=45, n_{c} / n_{e}=20, I_{16}=2$ and with assumed corona temperature $T_{e}=1$, we obtain $120 \mu \mathrm{m}$, close enough to 140 $\mu \mathrm{m}$ observed in Fig. 2. The observed values may have some error, since the observed timing is 200 ps after the main hole-boring pulse peak and the interference fringes may represent the self-focused hole after the time delay. The observed distance was slightly longer than that evaluated. This difference could be attributed to the plasma nonuniformity, since the estimation is for a uniform plasma and the selffocusing could take place more slowly for a higher plasma density.

It is known that the laser self-focusing could break up into filaments under certain conditions [12]. The self-focused laser could excite ion acoustic waves in the transverse direction of the propagation with a large growth rate of the ponderomotive filamentation instability. The transmitted light through this ion acoustic grating could be scattered into randomly the oriented direction with a certain scattered angle.

The most unstable mode is given by $k_{\perp}=1 / 2\left(\mathrm{v}_{\mathrm{osc}} /\right.$ $\left.\mathrm{v}_{e}\right)\left(\omega_{p e} / \omega_{0}\right)\left(\omega_{0} / c\right)$, where $k_{\perp}$ is the unstable wave number, $v_{\text {osc }}$ is the electron oscillating velocity in the laser field, $\mathrm{v}_{e}$ is the electron thermal velocity, $\omega_{p e}$ is the electron plasma frequency, $\omega_{0}$ is the laser frequency. The angle of dispersion is now expressed by

$$
\theta=1 / 2\left(\mathrm{v}_{\mathrm{osc}} / \mathrm{v}_{e}\right)\left(\omega_{p e} / \omega_{0}\right)
$$

This cone angle corresponds to $37^{\circ}$ at $3 \times 10^{20} \mathrm{~W} / \mathrm{cm}^{3}$ and at $200 \mu \mathrm{m}$ focus position (from the original target surface) in the preformed plasma. The plasma temperature is assumed to be $1 \mathrm{keV}$ and the laser intensities are assigned on the given preformed plasma density and laser focus geometrical cone. This implies that the laser could be spread within over 140 $\mu \mathrm{m}$ radius with many filaments near the target surface. Figures 3 and 5 show striated jets in the experiment and filamentation in the simulation for the third-harmonic drive. If this unstable mode is excited and the laser is spread into the cone given by Eq. (3), the filaments irradiate the $n_{c}$ surface and could result in many small high-density jets as a result of the very local heating of the filamentation with the striations in Fig. 3 in a 150-200 $\mu \mathrm{m}$ radius, consistent with the above understanding. We have used these numbers as a guide line for our interpretation, not as accurate estimation.

We have shown that a whole beam self-focusing can be created by a 1053-nm, 2-TW laser beam, while striations are observed for the case of a 351-nm laser beam. The 1053-nm case shows that there is a clear whole beam self-focusing sustained in the underdense region. The 351-nm case shows that there are many density striations created in the underdense region. 2(1/2) D PIC simulation results support the experimental results. The comparisons of the experiment and simulation lead to the conclusion that the striations are density jets as a result of that the focused 351-nm laser became unstable to break into filaments. The laser intensity dependence shown in the PIC simulation results indicate that the self-focus is initiated with the ponderomotive force effect, resulting in that the third-harmonic laser light needs at least ten times more energy to create a whole beam self-focusing than the infrared. 
We are indebted to Dr. M. Nakai and H. Azuma for their support with the uv interferometer. S. Nakaji and Y. Oshikane's earlier effort introduced the interferometer. We acknowledge the engineering support by Dr. M. Takagi, Y. Kimura, the laser operation group (GO), target fabrication group (T), and experimental support group (Mt). We are grateful to Professor S. Nakai for encouragement. We thank Professor A. Pukhov at ILE, Osaka, visiting from MPQ, Germany, for stimulating discussions and comments.
[1] K. A. Tanaka, B. Boswell, R. S. Craxton, L. M. Goldman, R. Short, and J. Soures, Phys. Fluids 28, 2910 (1985).

[2] P. E. Young, J. H. Hammer, S. C. Wilks, and W. Kruer, Phys. Plasmas 2, 2825 (1995).

[3] H. M. Milchberg, C. G. Durgee III, and J. Lynch, J. Opt. Soc. Am. A 12, 731 (1995).

[4] T. R. Clark and H. M. Milchbreg, Phys. Rev. Lett. 78, 2373 (1997).

[5] P. A. Norreys, M. Zepf, S. Moustazis, A. P. Fews, J. Zhang, P. Lee, M. Bakarezos, C. N. Dnason, and A. E. Dangor, Phys. Rev. Lett. 76, 1832 (1996).

[6] M. Tabak et al., Phys. Plasmas 1, 1626 (1994).

[7] P. E. Young, Phys. Plasmas 2, 2815 (1995).

[8] A. Pukhov and J. Meyer-ter-Vehn, Phys. Rev. Lett. 76, 3975 (1996); 79, 2686 (1997).

[9] J. Fuchs, G. Malka, J. C. Adam, F. Amiranoff, S. D. Baton, N. Blanchot, A. Heron, G. Laval, J. L. Miquiel, P. Mora, H.
Pepin, and C. Rosseaux, Phys. Rev. Lett. 80, 1658 (1998).

[10] K. Takahashi, R. Kodama, K. A. Tanaka H. Hashimoto, Y. Kato, K. Mima, F. Weber, T. Barbee, and L. DaSilva, ILE Research Report ILE 9811P (unpublished); R. Kodama, K. Takahashi, K. A. Tanaka, Y. Kato, K. Murai, F. Weber, T. W. Barbee, and L. B. DaSilva, Rev. Sci. Instrum. 70, 543 (1999).

[11] R. Kodama, K. Takahashi, K. A. Tanaka, M. Tsukamoto, H. Hashimoto, Y. Kato, and K. Mima, Phys. Rev. Lett. 77, 4906 (1996).

[12] S. Wilks, P. Young, J. Hammer, M. Tabak, and W. Kruer, Phys. Rev. Lett. 73, 2994 (1994).

[13] P. Young, M. E. Foord, J. H. Hammer, W. L. Kruer, M. Tabak, and S. C. Wilks, Phys. Rev. Lett. 75, 1082 (1995).

[14] V. Malka, E. De Wisperlaere, F. Amiranof, S. Baton, R. Bonadio, C. Coulaund, and R. Haroutunian, Phys. Rev. Lett. 79, 2979 (1997).

[15] C. Max, Phys. Fluids 19, 74 (1976). 